Bone is often the target of hormones, but it also acts as an endocrine organ, releasing a hormone that enhances sugar and fat metabolism. Boneforming cells secrete the protein osteocalcin, which acts as a hormone, increasing the mass of insulin-producing pancreatic cells, serum insulin levels and fat cell sensitivity to insulin. (Cell 130, 456-469)

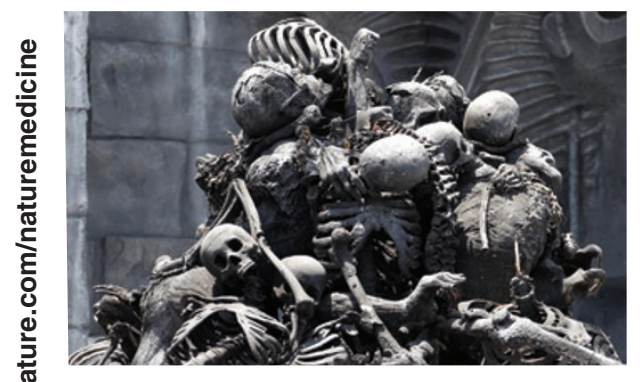

Attempts to derive stem cells that can be used for regenerative therapy without destroying embryos continued unabated this year. In January, researchers showed that cells present 으 in amniotic fluid can differentiate into a wide variety of cell types. In June, two independent groups reported that mouse fibroblasts can be reprogrammed into cells that closely resemble embryonic stem cells, and in September, another group identified a new marker for multipotent stem cells in the testes that may enable the use of these cells for therapy. (Nat. Biotechnol.25, 100-106, Nature 448, 313-317; 318-324; 449, 346-350)

- Lung cancers, including the poorly understood tumors associated with smoking, may be triggered by the fusion of two genes, Japanese researchers reported in August. Formed by a small chromosomal inversion, the transforming gene fusion generates an activated kinase, and is found in seven percent of tumors from smokers and nonsmokers. Small compounds already known to inhibit the kinase may be able to treat the disease. (Nature 448, 561-566)



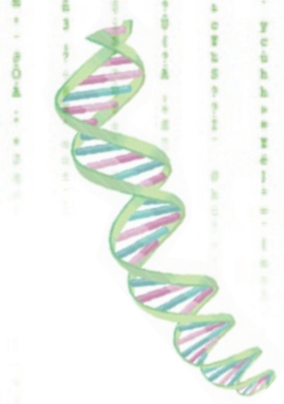

- Remember the old wives' tale that carrots can help you see in the dark? A cluster of papers provided a sounder reason to get your daily dose of vitamin A: it may help ward off inflammatory bowel disease by promoting the generation of regulatory $\mathrm{T}$ cells, which dampen inflammation. Dendritic cells in the gut are particularly adept at synthesizing the vitamin A metabolite retinoic acid, which, along with TGF- $\beta$, skews T cells toward becoming regulatory $\mathrm{T}$ cells. (Science 317 , 256-260; J. Exp. Med. 204, 1757-1764; 1765-1774; 1775-1785)

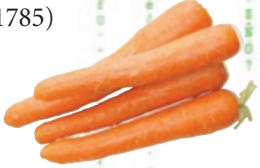

- Eating too much can lead to increased inflammation in fat tissue and, over time, trigger diabetes. In May, researchers showed that STAMP2, a protein in fat cells, controls proper nutrient storage while keeping inflammation at bay. Feeding increases the levels of STAMP2, reducing the expression of key cytokines that promote inflammation and stimulating the cell's insulin signaling pathway, which regulates the storage of excess nutrients. (Cell 129, 537-548)

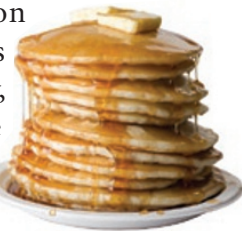

- A flurry of papers this year unveiled the importance of microRNAs (miRNAs) in heart development, function and disease. The studies delineated the mechanisms by which specific miRNAs act to regulate heart morphogenesis, contractility, electrical conduction and remodeling. Hearts from patients with cardiomyopathy or coronary artery disease have abnormal levels of some of these miRNAs, suggesting that they could be new therapeutic targets for heart disease. (Nat. Med. 13, 486-491; 613-618; Cell 129, 303-317; Science 316, 575-579)

\section{Notable advances}

Some of the key papers published in 2007
- How the heart is put together during embryonic develópment has been extensively studied, but scientists reported a new twist to this story late last year. Three groups isolated mouse multipotent progenitor cells that can give rise to three major heart cell types - cardiac muscle, smooth muscle and endothelial cells. The findings may help researchers devise treatments for congenital and adult heart disease. (Dev. Cell 11; 723-732; Cell 127, 1137-1150; 1151-1165)

- Platelets live for only ten days before they are removed from the bloodstream. In March, an Australian team revealed that anuclear apoptosis is the secret to this platelet lifespan. Antagonism between the proapoptotic protein Bak and the antiapoptotic $\mathrm{Bcl}-\mathrm{x}_{\mathrm{L}}$ protein sets up the ticking clock, and targeting this interaction could extend or limit the life of platelets to maintain healthy platelet counts. (Cell 128, 1173-1186)

MiRNAs are key in the battle between viruses and their host cells, as revealed by four papers this year. Host cells produce a battery of miRNAs that shut off HIV gene expression, suppressing replication of the virus and contributing to latent infection. But other viruses can make their own miRNAs to hit back: human cytomegalovirus expresses miRNAs that promote the survival of infected cells. (Science 315, 1579-1582; 317, 376-381; 316, 1345-1348; Nat. Med. 13, 1241-1247)

- The decades-long search for genes associated with multiple sclerosis finally bore fruit this year, as three groups reported a link between polymorphisms in the interleukin-7 receptor gene and the disorder. The polymorphisms may dampen the signaling pathways downstream of interleukin-7, potentially affecting the survival of some inflammatory cells. (Nat. Genet. 39, 1083-1091; 1108-1113; N. Engl. J. Med. 357, 851-862)

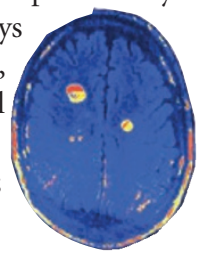

\title{
Running the rat (lab) race
}

3.803 Number of doctoral degrees awarded in the

3,803

6,368

$70.3 \%$ biological sciences in the US in 1981

Number of doctoral degrees awarded in the

biological sciences in the US in 2005

Researchers in 1981 with tenure or tenure-track

positions four to seven years after their PhDs
$53.7 \%$

37

42
Researchers in 2003 with tenure or tenure-track positions four to seven years after their PhDs Average age in 1980 of researchers receiving their first US National Institutes of Health RO1 grant Average age in 2004 of researchers receiving their first R01 grant 